\title{
Modeling and Verification of Pipelined Embedded Processors in the Presence of Hazards and Exceptions
}

\author{
Prabhat Mishra, Nikil Dutt \\ Architectures and Compilers for Embedded Systems (ACES), Center for Embedded Computer \\ Systems, University of California, Irvine, CA 92697, USA
}

\begin{abstract}
Embedded systems present a tremendous opportunity to customize designs by exploiting the application behavior. Due to increasing design complexity deeply pipelined high performance embedded processors are common today. In the presence of hazards and exceptions the validation of pipelined embedded processors is a major challenge. We extend a Finite State Machine (FSM) based modeling of pipelined processors to verify the pipeline specification in the presence of hazards and multiple exceptions. Our approach leverages the system architect's knowledge about the behavior of the pipelined processor, through Architecture Description Language (ADL) constructs, and thus allows a powerful top-down approach to pipeline verification. We applied this methodology to the DLX processor to demonstrate the usefulness of our approach
\end{abstract}

Key words: Pipeline Verification, Architecture Description Language.

\section{INTRODUCTION}

Verification is a major challenge in design and development of programmable embedded systems. It is especially difficult for deeply pipelined high performance processors in the presence of hazards and multiple exceptions. System architects critically need modelling techniques that allow rapid exploration, customization and validation of different processor pipeline configurations, tuned for a specific application domain. Recent approaches on language-driven Design Space Exploration (DSE) [4, 7], use ADL to capture the processor architecture, generate automatically a software toolkit for that processor, and provide feedback to the designer on

The original version of this chapter was revised: The copyright line was incorrect. This has been corrected. The Erratum to this chapter is available at DOI: 10.1007/978-0-387-35599-3_29 
the quality of the architecture. While some of these approaches $[4,5,7]$ captures hazards and interrupts in ADL for specific types of architectures, to our knowledge no previous approach has an explicit way of describing hazards and interrupts for a wide variety of processor and memory architectures [6], and the attendant task of verifying the processor pipeline in the presence of hazards and multiple exceptions. It is important to verify the ADL description of the architecture to ensure the correctness of the software toolkit. The benefits of verification are two-fold. First, the process of any specification is error-prone and thus verification techniques can be used to check for correctness and consistency of specification. Second, changes made to the processor during DSE may result in incorrect execution of the system and verification techniques can be used to ensure correctness of the modified architecture.

The rest of the paper is organized as follows. Section 2 presents related work addressing verification of pipelined processors. Section 3 outlines our approach and the overall flow of our environment. Section 4 presents our FSM based modeling of processors in the presence of hazards and exceptions. Section 5 proposes our verification technique followed by a case study in Section 6. Section 7 concludes the paper.

\section{RELATED WORK}

Several approaches for formal or semi-formal verification of pipelined processors have been developed in the past. Theorem proving techniques, for example, have been successfully adapted to verify pipelined processors [2, $14,16]$. However, these approaches require a great deal of user intervention, especially for verifying control intensive designs. Burch and Dill presented a technique for formally verifying pipelined processor control circuitry [1]. This technique has been extended to handle more complex pipelined architectures by several researchers $[15,17]$. All the above techniques attempt to formally verify the implementation of pipelined processors by comparing the pipelined implementation with its sequential (ISA) specification model, or by deriving the sequential model from the implementation. On the other hand, in our verification approach, we define a set of properties, and verify the correctness of pipelined processors by testing whether the properties are satisfied using a FSM-based model.

An architecture description language (ADL) driven modeling and validation technique is presented in [11]. Mishra et al. [9] presented an FSM based modeling of pipelined processors with in-order execution and is closest to our approach. Our work extends this model to verify the processor pipeline in the presence of hazards and multiple exceptions. 


\section{OUR APPROACH}

Figure 1 shows the modeling and validation flow. In our IP library based exploration and verification scenario, the designer starts by specifying the processor description in an ADL. The FSM model of the pipelined processor description is automatically generated from this ADL description. We have defined several properties such as determinism, in-order execution, and finiteness, to ensure that the ADL description of the architecture is wellformed in the presence of hazards and multiple exceptions. Our automatic property checking framework determines if all the necessary properties are satisfied or not. In case of failure, it generates traces so that designer can modify the ADL specification of the architecture. If the verification is successful, the software toolkit (including compiler and simulator) can be generated for design space exploration.

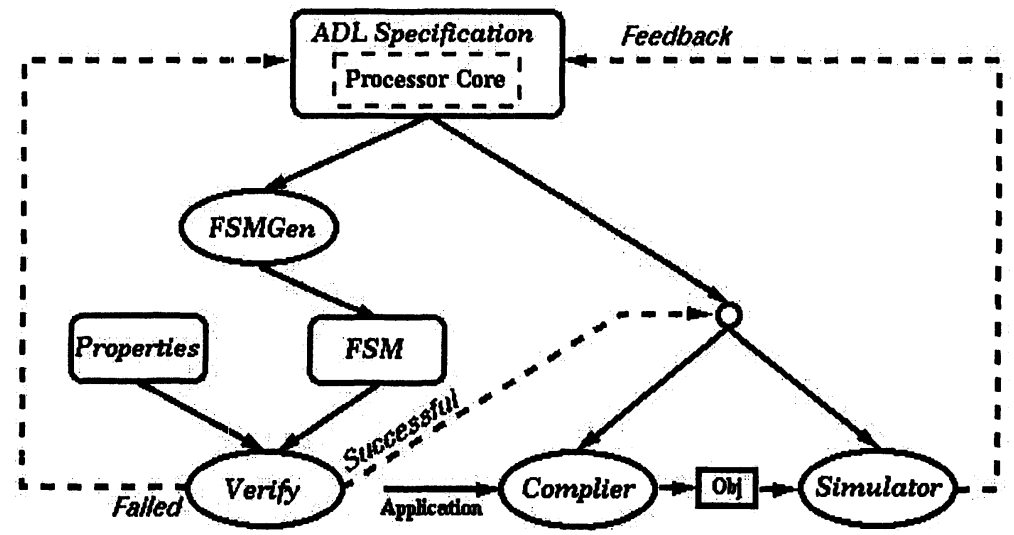

Figure 1. The flow in our approach

\section{MODELING OF PROCESSOR PIPELINES}

In this section we describe how we derive the FSM model of the pipeline from the ADL description of the processor. We first explain how we specify hazards, stalls, exceptions, interrupts, and flow conditions in ADL, then we present the FSM model of the processor pipeline using the information captured in the ADL. The detailed description can be found in [13].

\subsection{Processor Pipeline Description in ADL}

We have chosen the EXPRESSION ADL [8] that captures the structure, behavior, and the mapping between them for the processor pipeline. The $\mathrm{ADL}$ description can be used to detect hazards. Structural hazards are 
captured using reservation tables [3]. Using the same constraint information (regarding sources and destinations) available in RTs the data hazards viz., read-after-write (RAW), write-after-write (WAW), and write-after-read (WAR), can be detected. Control hazards due to branches can have different outcomes depending on how the branch is handled for that architecture. In our framework we treat branch mis-prediction as an exception and the necessary flushing can happen during exception handling.

A functional unit can be stalled due to external signals or due to conditions arising inside the processor pipeline. For units, with multiple children the stalling conditions due to internal contribution may differ. For example, the unit $\mathrm{UNIT}_{\mathrm{i}-1, \mathrm{j}}$ in Figure 2 with $q$ children can be stalled when any one of its children is stalled, or when some of its children are stalled, or when all of its children are stalled; or when none of its children are stalled. During specification, designer selects from the set (ANY, SOME, ALL, NONE) the internal contribution along with any external signals to specify stall condition for each unit.

A unit is in normal flow if it can receive instruction from its parent unit and can send it to its child unit. Typically, a unit performs nop insertion when it does not receive any instruction from its parent and its child unit is not stalled. The Program Counter (PC) unit can be stalled due to external signals such as cache miss or when the fetch unit is stalled. When a branch is taken the PC unit is said to be in branch taken state. The PC unit is in sequential execution mode when the fetch unit is in normal flow, there are no external interrupts, and the current instruction is not a branch instruction. A unit is in exception condition when it receives an exception or due to internal contribution. We specify exceptions and interrupts in ADL [13].

\subsection{FSM Model of Processor Pipelines}

This section presents an FSM-based modeling of controllers in pipelined processors. Figure 2 shows a fragment of a processor pipeline. The oval boxes represent units, rectangular boxes represent pipeline latches, and arrows represent pipeline edges. We assume a pipelined processor with inorder execution as the target for modeling and verification. The pipeline consists of $N$ stages. Each stage can have more than one pipeline register. In this paper we call these pipeline registers instruction registers (IR). Let Stage $_{i}$ denote the $i$-th stage where $(0 \leq \mathrm{i} \leq \mathrm{N}-1)$, and $\mathrm{N}_{\mathrm{i}}$ the number of pipeline registers between Stage $\mathrm{i}_{\mathrm{i}-1}$ and Stage $e_{i}$. Let $I R_{i, j}$ denotes an instruction register between Stage $_{\mathrm{i}-1}$ and $\operatorname{Stage}_{\mathrm{i}}\left(1 \leq \mathrm{j} \leq \mathrm{N}_{\mathrm{i}}\right)$. During execution the instruction stored in $\mathbb{I R}_{\mathrm{i}, \mathrm{j}}$ is executed at Stage $\mathrm{i}_{\mathrm{i}}$ and then stored into the next instruction register $\mathbb{R}_{\mathrm{i}+1, \mathrm{~s}+\mathrm{k}}(1 \leq \mathrm{k} \leq \mathrm{q})$. In this paper, we define a state of the $\mathrm{N}$-stage pipeline as values of $\mathrm{PC}$ and $(\mathrm{N}-1) \times \sum_{\mathrm{i}=1}^{\mathrm{N}-1} \mathrm{~N}_{\mathrm{i}}$ instruction registers. 
Let $P C(t)$ and $\mathbb{R}_{i, j}(t)$ denote the values of $P C$ and $\mathbb{R}_{i, j}$ at time $t$, respectively. Then, the state of the pipeline at time $t$ is defined as

$$
S(t)=<P C(t), I R_{1,1}(t), \ldots \ldots, I R_{N}-1, N_{N}-1(t)>
$$

We first describe the conditions for exception (XN), stalling (ST), normal flow (NF), nop insertion (NI), sequential execution (SE), and branch taken (BT) in the FSM model, then we describe the state transition functions possible in the FSM model using these conditions.

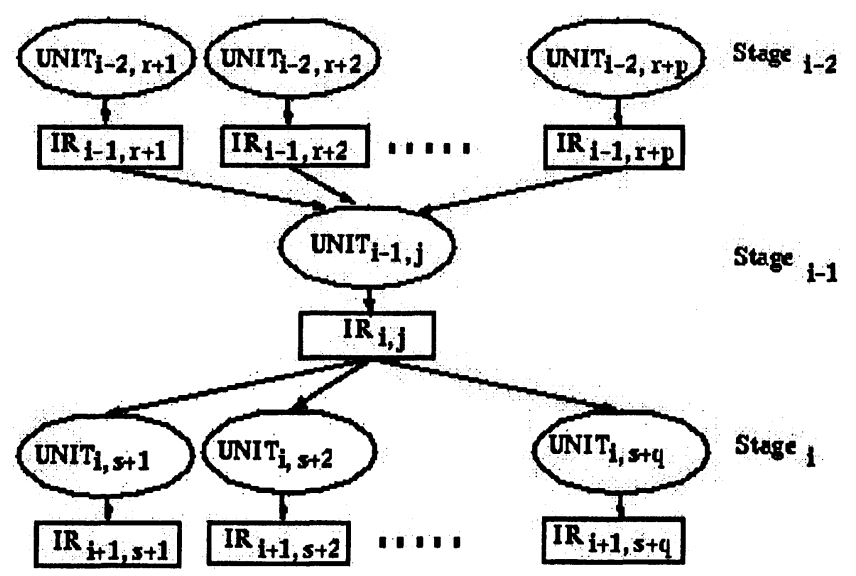

Figure 2. A fragment of a processor pipeline

\subsubsection{Modeling Conditions in FSM}

Let us assume, every instruction register $\mathbb{R}_{\mathrm{i}, \mathrm{j}}$ has an exception bit $X \mathrm{~N}_{\mathbb{I R} \mathrm{i}, \mathrm{j}}$, which is set when the exception condition (cond ${ }^{X N}{ }_{I R, j}$ say) is true. $\mathrm{XN}_{\mathbb{I R}_{i, j}}$ has two components as shown in Equation 2. Similarly, the conditions for stall, normal flow, nop insertion (for instruction registers), sequential execution, and branch taken (for $\mathrm{PC}$ ) are shown in Equation 3-8. Let us assume, $\mathrm{BT}_{\mathrm{PC}}$ bit is set when the unit completes execution of a branch instruction.

$$
\begin{aligned}
& \text { cond }_{I R_{i j}}^{X N}=X N_{I R_{i j}}=X N_{I R_{i j}}^{\text {child }}+X N_{I R_{i j}}^{\text {self }} \\
& \text { cond }_{I R_{i j}}^{S T}=S T_{I R_{i j}} \cdot \overline{X N_{I R_{i j}}}=\left(S T_{I R_{i j}}^{\text {child }}+S T_{I R_{i j}}^{\text {self }}\right) \overline{X N_{I R_{i j}}} \\
& \operatorname{cond}_{I R_{i j}}^{N F}=N F_{I R_{i j}}^{\text {parent }} \cdot N F_{I R_{i j}}^{\text {child }} \cdot \overline{S T_{I R_{i j}}^{\text {self }}} \cdot \overline{X N_{I R_{i j}}} \\
& \text { cond }_{I R_{i j}}^{N I}=N I_{I R_{i j}}^{\text {parent }} \cdot N I_{I R_{i j}}^{\text {child }} \cdot \overline{S T_{I R_{i j}}^{\text {self }}} \cdot \overline{X N_{I R_{i j}}} \\
& \text { cond }_{P C}^{S E}=N F_{P C}^{\text {child }} \cdot \overline{S T_{P C}^{\text {self }}} \cdot \overline{B T_{P C}} \cdot \overline{X N_{I R_{1 j}}} \\
& \text { cond }_{P C}^{S T}=\left(S T_{P C}^{\text {child }}+S T_{P C}^{\text {self }}\right) \cdot \overline{B T_{P C}} \cdot \overline{X N_{I R_{1 j}}} \\
& \text { cond }_{P C}^{B T}=B T_{P C}+X N_{I R_{1 j}}
\end{aligned}
$$




\subsubsection{Modeling State Transition Functions}

In this section, we describe the next state function of the FSM. Let $\mathrm{f}^{\mathrm{NS}}$ and $\mathrm{f}^{\mathrm{NS}}{ }_{\mathbb{R} \mathrm{ij}}\left(1 \leq \mathrm{i} \leq \mathrm{N}-1,1 \leq \mathrm{j} \leq \mathrm{N}_{\mathrm{i}}\right)$ denote next state functions for $\mathrm{PC}$ and $\mathbb{I R}_{\mathrm{i}, \mathrm{j}}$ respectively. For $\mathrm{PC}$, we define three types of state transitions as follows.

$$
P C(t+1)=f_{P C}^{N S}(S(t), I(t))=\left\{\begin{array}{cl}
P C(t)+L & \text { if } \quad \operatorname{cond}_{P C}^{S E}(S(t), I(t))=1 \\
t \arg \text { et } & \text { if } \operatorname{cond}_{P C}^{B T}(S(t), I(t))=1 \\
P C(t) & \text { if } \operatorname{cond}_{P C}^{S T}(S(t), I(t))=1
\end{array}\right.
$$

Here, $\mathrm{I}(\mathrm{t})$ represents a set of external signals at time $\mathrm{t}, L$ represents the instruction length, and target represents the branch target address, which is computed at a certain pipeline stage. Similarly, for the instruction registers, $I_{\mathrm{i}, \mathrm{j}}$, we define four types of state transitions as follows.

$$
I R_{i, j}(t+1)=f_{I R_{i, j}}^{N S}(S(t), I(t))=\left\{\begin{array}{cll}
I R_{i-1, r+l}(t) & \text { if } & \operatorname{cond}_{I R_{i j}}^{N F}(S(t), I(t))=1 \\
I R_{i, j}(t) & \text { if } & \operatorname{cond}_{I R_{i j}}^{S T}(S(t), I(t))=1 \\
n o p & \text { if } \operatorname{cond}_{I R_{i j}}^{N I}(S(t), I(t))=1 \\
n o p & \text { if } & \operatorname{cond}_{I R_{i j}}^{X N}(S(t), I(t))=1
\end{array}\right.
$$

In the above formulas, nop denotes a special instruction indicating that there is no instruction in the instruction register. For the first instruction register the data comes from the instruction memory pointed by the PC.

\section{VERIFICATION OF IN-ORDER EXECUTION}

Based on the FSM modeling presented in Section 4, we propose a method to verify the correctness of pipeline controllers with in-order execution. A pipelined processor with in-order execution is correct if all instructions, which are fetched from instruction memory flow from the first stage to the last stage while maintaining their execution order. In this section we describe the properties needed for verifying the in-order execution.

\subsection{Determinism Property}

The next state functions for all state registers must be deterministic. This property is valid if all the following equations (Equation 11-14) hold. The first two equations mean that, in the next state function for each state register, the flow conditions must cover all possible combinations of 
processor states $\mathrm{S}(\mathrm{t})$ and external signals $\mathrm{I}(\mathrm{t})$. The last two guarantee that any two conditions are disjoint for each next state function. Informally, exactly one of the conditions should be true in a cycle for each state register.

$$
\begin{gathered}
\operatorname{cond}_{P C}^{S E}+\operatorname{cond}_{P C}^{B T}+\operatorname{cond}_{P C}^{S T}=1 \\
\operatorname{cond}_{I R_{i, j}}^{N F}+\operatorname{cond}_{I R_{i, j}}^{S T}+\operatorname{cond}_{I R_{i j}}^{N I}+\operatorname{cond}_{I R_{i j}}^{X N}=1 \\
\forall x, y(x, y \in\{S E, B T, S T\} \cap x \neq y) \quad \operatorname{cond}_{P C}^{x} \cdot \operatorname{cond}_{P C}^{y}=0 \\
\forall x, y(x, y \in\{N F, S T, N I, X N\} \cap x \neq y) \quad \operatorname{cond}_{I R_{i j}}^{x} \cdot \operatorname{cond}_{I R_{i j}}^{y}=0
\end{gathered}
$$

\subsection{In-Order Execution Property}

In order to guarantee in-order execution, state transitions of adjacent instruction registers must depend on each other. All illegal state transitions of adjacent pipeline stages are described below using Figure 2. An instruction register cannot be in normal flow if all the parent instruction registers (adjacent ones) are stalled. If such a combination of state transitions are allowed, the instruction stored in $\mathbb{R}_{\mathrm{i}-1, \mathrm{r}+1}(1 \leq 1 \leq \mathrm{p})$ at time $\mathrm{t}$ will be duplicated, and stored into both $\mathbb{R}_{\mathrm{i}-1, \mathrm{r}+1}$ and $\mathbb{I R}_{\mathrm{i}, \mathrm{j}}$ at the next cycle. Therefore, the instruction will be executed more than once. More formally, the Equation 15 should be satisfied.

$$
1 \mathrm{l}_{l=1} \operatorname{cona}_{I R_{i-1, \mathrm{r}+l}} \cdot \operatorname{cona}_{I R_{i, j}}=\mathrm{U}
$$

Similarly, if $\mathbb{R}_{\mathrm{i}, \mathrm{j}}$ flows normally, at least one of its child latches should also flow normally. If all of its child latches are stalled, the instruction stored in $\mathbb{R}_{\mathrm{i}, \mathrm{j}}$ disappears. The Equation 16 should be satisfied. Similarly, if $\mathbb{I R}_{\mathrm{i}, \mathrm{j}}$ is in nop insertion, at least one of its child latches should not be stalled. The Equation 17 should be satisfied. Similarly, an instruction register cannot be in nop insertion, if previous instruction register is in normal flow. The Equation 18 should be satisfied.

$$
\begin{gathered}
\operatorname{cond}_{I R i, j}^{N F} \cdot \bigcap_{k=1}^{q} \operatorname{cond}_{I R_{i+1, s+k}}^{S T}=0 \\
\operatorname{cond}_{I R i, j}^{N I} \cdot \bigcap_{k=1}^{q} \operatorname{cond}_{I R_{i+1, s+k}}^{S T}=0 \\
\operatorname{cond}_{I R_{i-1, r+1}}^{N F} \cdot \operatorname{cond}_{I R i, j}^{N I}=0 \\
\operatorname{cond}_{I R_{t-1, r+1}}^{N I} \cdot \operatorname{cond}_{I R i, j}^{N I}=0 \\
\operatorname{cond}_{I R_{i-1, r+1}}^{N I} \cdot \operatorname{cond}_{I R i, j}^{X N}=0 \\
\operatorname{cond}_{I R_{i-1, r+1}}^{S T} \cdot \operatorname{cond}_{I R i, j}^{X N}=0 \\
\operatorname{cond}_{I R_{i-1, r+1}}^{N I} \cdot \operatorname{cond}_{I R i, j}^{X N}=0
\end{gathered}
$$

$$
\begin{aligned}
& \text { (16) } \quad \operatorname{cond}_{P C}^{S T} \cdot \operatorname{cond}_{I R_{1, j}}^{N F}=0 \\
& \text { (17) } \quad \operatorname{cond}_{P C}^{S E} \cdot \bigcap_{j=1}^{N_{1}} \operatorname{cond}_{I R_{1, j}}^{S T}=0 \\
& (18) \quad \operatorname{cond}_{P C}^{B T} \cdot \bigcap_{j=1}^{N_{1}} \operatorname{cond}_{I R_{1, j}}^{S T}=0 \\
& (19) \quad \operatorname{cond}_{P C}^{S E} \cdot \operatorname{cond}_{I R_{1, j}}^{N I}=0 \\
& (20) \quad \operatorname{cond}_{P C}^{B T} \cdot \operatorname{cond}_{I R_{1, j}}^{N I}=0 \\
& (21) \quad \operatorname{cond}_{P C}^{S E} \cdot \operatorname{cond}_{I R_{1, j}}^{X N}=0 \\
& (22) \quad \operatorname{cond}_{P C}^{S T} \cdot \operatorname{cond}_{I R_{1, j}}^{X N}=0
\end{aligned}
$$


Similarly, an instruction register cannot be in nop insertion, if previous instruction register is also in nop insertion. The Equation 19 should be satisfied. Finally, an instruction register cannot be in normal flow, stall, or nop insertion if next (child) instruction register is in exception. The Equation 20-22 should be satisfied. Similarly, the state transition of PC must depend on the state transition of $\mathbb{I R}_{1, j}$ as shown in Equation 23-29. All the equations (Equation 15-29) must hold to ensure correct in-order execution.

\section{A CASE STUDY}

In a case study we successfully applied the proposed methodology to the single-issue DLX [10] processor. We have chosen DLX processor since it has been well studied in academia and has few interesting features viz., fragmented pipelines, multicycle units etc. The DLX processor has five pipeline stages viz., Fetch (IF), Decode (ID), Execute, Memory (MEM), and WriteBack (WB). The Execute stage consists of four parallel execution paths viz., integer ALU (EX), seven-stage multiplier (M1-M7), four-stage floating-point adder (A1-A4) and a multicycle division unit (DIV).

We used the EXPRESSION ADL [8] to capture the structure and behavior of the DLX processor. We captured the conditions for exception, stalling, normal flow, branch taken and nop insertion in the ADL [12]. Using the ADL description, we automatically generated the equations for flow conditions for all the units [12]. The necessary equations for verifying the properties viz., determinism, in-order execution etc., are generated automatically from the given ADL description. The Eqntott tool converts these equations in two-level representation of a two-valued Boolean function. We have used Espresso to minimize the equations. These minimized equations are analyzed to verify whether the properties are violated or not. The complete verification took 45 seconds on a $333 \mathrm{MHz}$ Sun Ultra-5 with 128M RAM.

Our framework determined that the in-order execution is violated in the presence of an exception in A2 stage of the floating-point adder and generated a simple instruction sequence which violates in-order execution: floating-point addition followed by integer addition. The decode unit issued floating point addition $\mathrm{I}_{\text {fadd }}$ operation in cycle $n$ to floating-point adder pipeline (A1 - A4) and an integer addition operation $\mathrm{I}_{\mathrm{iadd}}$ to integer ALU (EX) at cycle $n+1$. Due to the exception in A2 stage and our exception model the $\mathrm{A} 1, \mathrm{ID}$ and $\mathrm{IF}$ stages got flushed whereas the $\mathrm{I}_{\mathrm{iadd}}$ operation continued its execution.

We modified the ADL description to incorporate the notion of in-order relationship among units. For example, for EX unit the in-order children are 
M2 and A2. Note that, when M3 stage is in exception condition, the M2 stage and it's parent (normal parent M1 and in-order parents EX and A1) stages will be flushed. Similarly, the in-order child for M2 stage is A2 and so on. However, this modeling is not good enough in the presence of multicycle functional units (e.g., DIV unit). The in-order children (parent) information will change depending on how many cycles the division operation is in DIV unit. So we model division unit with $\mathrm{n}$-stages $\left(\mathrm{D}_{1}, \mathrm{D}_{2}, \ldots, \mathrm{D}_{\mathrm{n}}\right)$, where $n$ is the latency of the division operation, with the assumption that the stage $D_{i}$ will have the division operation after $i$ cycles and only one stage will have a valid operation at a time. Now we can extend the in-order child concept for multicycle units as well. Now, the in-order children for EX unit are M2, A2 and D2. Similarly, the in-order children for D1 unit are A2 and M2. Since we are considering single-issue machine, two units in the same level will not have valid instruction at the same point in time. For example, M1 and A1 cannot have valid instructions at the same point in time. The in-order execution was successful for this modeling in the presence of a single exception. This modeling is sufficient to handle multiple exceptions where the exception closer to completion has the higher priority. This is due to the fact that the exception closer to completion will flush all the operations above and thereby masks all the exceptions generated at the earlier stages in the pipeline. For example, if there are exceptions in $\mathrm{M} 3$ and $\mathrm{A} 1$, the exception in M3 will mask the exception in A1 stage. However, this modeling will not work when the exceptions have a fixed priority. If the exception in A1 has higher priority than the exception in M3 and if the current modeling is used, the exception in M3 will be incorrectly selected. To solve this problem we modeled the interrupt handler as described in [13]. Only one interrupt will be selected at a time. Hence, in the presence of fixed priority based multiple exceptions the DLX processor can be verified.

\section{CONCLUSION}

Embedded systems present a tremendous opportunity to customize designs by exploiting the application behavior. A major challenge has been to efficiently model and verify the complex pipeline behavior during the rapid exploration and customization of embedded systems. This paper proposed an ADL driven modeling and verification technique for pipelined embedded processors in the presence of hazards and multiple exceptions. The FSM model of the pipeline controller is generated automatically from this modeling. Based on the modeling we presented a set of properties that are used in our framework to verify the correctness of the in-order execution of the pipeline. We used the DLX processor as an example to demonstrate 
the usefulness of our approach. We are extending our modeling and verification technique towards VLIW and superscalar processors.

\section{ACKNOWLEDGMENTS}

This work was partially supported by grants from DARPA(F33615-00-C1632), Hitachi Ltd., and Motorola Inc. We would like to acknowledge Dr. Hiroyuki Tomiyama for his contribution to the validation work.

\section{REFERENCES}

[1] J. Burch and D. Dill. Automatic Verification of Pipelined Microprocessor Control. CAV, 1994.

[2] D. Cyrluk. Microprocessor Verification in PVS: A Methodology and Simple Example. Technical Report, SRI-CSL-93-12, 1993.

[3] P. Grun et al. RTGEN: An Algorithm for Automatic Generation of Reservation Tables from Architectural Descriptions. ISSS, 1999.

[4] C. Siska. A Processor Description Language Supporting Retargetable Multi-pipeline DSP Program Development Tools. ISSS, 1998.

[5] M. Freericks. The nML Machine Description Formalism. TR SM-IMP/DIST/08, TU Berlin CS Dept., 1993.

[6] P. Mishra et al. Functional Abstraction driven Design Space Exploration of Heterogeneous Programmable Architectures. ISSS, pages 256-261, 2001.

[7] V. Zivojnovic et al. LISA - machine description language and generic machine model for HW/SW co-design. IEEE Workshop on VLSI Signal Processing, 1996.

[8] A. Halambi et al. EXPRESSION: A language for architecture exploration through compiler/simulator retargetability. DATE, 1999.

[9] P. Mishra et al. Automatic Verification of In-Order Execution in Microprocessors with Fragmented Pipelines and Multicycle Functional Units. DATE, pages 36--43, 2002.

[10] J. Hennessy and D. Patterson. Computer Architecture: A quantitative approach. Morgan Kaufmann Publishers Inc, San Mateo, CA, 1990.

[11] P. Mishra et al. Automatic Modeling and Validation of Pipeline Specifications driven by an Architecture Description Language. ASP-DAC/VLSI Design, pages 458--463, 2002.

[12] P. Mishra et al. Architecture description language driven verification of in-order execution in pipelined processors. UCI-ICS TR 01-20, UC, Irvine, 2001.

[13] P. Mishra et al. Specification of Hazards, Stalls, Interrupts, and Exceptions in EXPRESSION. UCI-ICS Technical Report 01-05, University of California, Irvine, 2001.

[14] J. Sawada and J. W.A. Hunt. Trace table based approach for pipelined microprocessor verification. CAV, 1997.

[15] J. Skakkebaek, R. Jones, and D. Dill. Formal verification of out-of-order execution using incremental flushing. CAV, 1998.

[16] M. Srivas and M. Bickford. Formal verification of a pipelined microprocessor. IEEE Software; 1990; 7(5):52--64.

[17] M. Velev and R. Bryant. Formal verification of superscalar microprocessors with multicycle functional units, exceptions, and branch prediction. DAC, 2000. 